\title{
Theories and experiments in medical research
}

\section{F Passariello 1}

${ }^{1}$ Fondazione Vasculab ONLUS, via Francesco Cilea 280 - 80127 Naples, Italy

submitted: Sep 26, 2017, accepted: Sep 26, 2017, EPub Ahead of Print: Sep 26, 2017, published: Sep 27, 2017

Conflict of interest: None

DOI: 10.24019/jtavr.18 - Corresponding author: Dr. Fausto Passariello, afunzionale@tiscalinet.it

(C) 2017 Fondazione Vasculab impresa sociale ONLUS. All rights reserved.

Theoretical medical research is often considered marginal, in comparison with the universally accepted experimental research.

Medical journals as well as many meeting organizations, with the exception of specialized theoretical journals, nowadays prefer scientific contributes, which fit the pre-requisite of the well-known $\mathrm{IMRaD}^{1}$ sequence: introduction, aims, materials and methods, results, discussion and conclusion. Theoretical research and model design generally do not comply with this strict scheme, asking instead for more relaxed constraints.

The current issue of JTAVR guests 3 papers, which are just free-format examples of theoretical frames, able to explain several pathophysiological events in the venous system.

The $1^{\text {st }}$ (C Franceschi) deals with the problem of the 0 -point in pressure measurements, underlining the role of collapsible veins in physiology, laboratory sets and daily clinics. Indeed, the central venous pressure is usually monitored in intensive care units (ICUs), almost everywhere in the world, as well as it is part of many invasive hemodynamic research protocols.

The $2^{\text {nd }}$ (F Passariello) formulates the hypothesis that the venous collateral circulation, in addition to its beneficial effect by-passing a venous occlusion, has also a detrimental effect on the tissues it passes through, owing to the transport of catabolites and carbon dioxide.

The $3^{\text {rd }}$ (F Schelling) is the first paper ever published (1982) on the venous origin of multiple sclerosis and appears here as an authorized reprint, together with several notes. Looking at the wide resonance of this paper, made by a great deal of theoretical an experimental research works and hints to therapy, we understand something more about the role of theories as suggestions to research.

Generally theories must explain already accepted experimental results, but their main value is related to their ability to suggest new experimental research work, in order to corroborate or falsify their forecasts.

Franceschi provides new theoretical frame and tests it with a do-it-yourself set, built in the kitchen and described in a movie, which faces many cliché prejudices and asking for a deeper understanding of the concept of pressure.

Passariello asks for deeper studies on venous collateral circulation and for an analysis of the relationship between clinical severity and quantitative/categorized intensity of collaterality.

Schelling would greatly appreciate measurements of venous pressure impact on brain and spinal cord structures, especially during rapid body movements and taking into account physical constraints which limit the movement, mainly in the spinal cord.

Note that all these three papers can be related to venous research in multiple sclerosis, thus suggesting how to proceed in order to get new meaningful results.

This short "excursus" in theoretical research, well matching the aims of the Journal, shows how a structured (but not too strict) standard scheme is needed for a correct approach to hypotheses in research work.

Fausto Passariello

Editor in Chief 
F Passariello - Theories and experiments in medical research

\section{References}

1) International Committee of Medical Journal Editors.

of scholarly work in medical journals. http://www.icmje.org/icmjeRecommendations for the conduct, reporting, editing, and publication recommendations.pdf (2016, accessed April 2017). 